\title{
Problem Solving Style, Perceived Burdensomeness and Suicide Ideation among College Students
}

\author{
Akansha Gautam ${ }^{1} *$
}

\section{ABSTRACT}

Objectives: Recent evidence suggests that suicidal ideation is increasing among college going students, it is essential to upsurge our knowledge concerning the etiology of suicidal ideation among students. This study was conducted to examine the relationships between problemsolving skills appraisal, perceived burdensomeness, and suicidal ideation among University students. In addition, this study was conducted to examine problem-solving skills appraisal (including the three components: problem-solving confidence, approach-avoidance style, and personal control of emotion) and perceived burdensomeness as predictors of suicidal ideation. Methods: The participants consisted of 350 students from NCR located Universities. Results: Regression analysis estimated that undergraduate students with avoiding style, poor problemsolving confidence, limited personal control of emotion and perceived burdensomeness were associated with higher suicidal ideation. Problem-solving skills appraisal (including the three components of problem-solving confidence, approach-avoidance style, and personal control of emotion) and perceived burdensomeness predicted the suicidal ideation. Conclusion These findings underline the importance of studying contributing processes that explain how problem solving and perceived burdensomeness affects suicidal ideation.

Keywords: Problem- Solving Skills Appraisal, Perceived Burdensomeness, Suicidal Ideation

Suicide is one of the serious and an increasing public health problem faced all over the world. Amongst the other causes of death, it is the third highest cause in the entire world especially in the age group of 16 to 24years. Similarly, suicidal ideation is common during adolescence (National Center for Injury Prevention and Control 2010). According to the World Health Organization (WHO), one million people have died from suicide, and one suicidal death occurs every 40 seconds. WHO (2012) has also predicted that by the year 2020, one person every 20 seconds will die due to suicide if urgent action is not taken. Recent evidence suggests that

\footnotetext{
${ }^{1}$ Scientist 'D', Directorate of Aerospace Safety, Air HQ, RK Puram, Delhi, India *Responding Author

(C) 2016 I A Gautam; licensee IJIP. This is an Open Access Research distributed under the terms of the Creative Commons Attribution License (http://creativecommons.org/licenses/by/2.0), which permits unrestricted use, distribution, and reproduction in any Medium, provided the original work is properly cited.
} 


\section{Problem Solving Style, Perceived Burdensomeness and Suicide Ideation among College Students}

suicidal ideation and attempts have increased in Asian countries among university students, indicating this group may be an, at-risk population (Garlow et al., 2008).

Students undergo major and rapid transition during university years where in he/she moves out from a protected homely environment to a more competitive, independent and responsible environment. There is a major shift in social and psychological aspects in the students' lives, and they may experience different levels of difficulties through peer and academic pressures, occupational choices, and life goal decisions. With regard to the above-mentioned reasons, suicidal ideation is on the increase among University students, making it important to identify early-warning signs to prevent suicide. Therefore, this study was conducted to understand suicidal ideation and the variables associated with suicidal ideation among undergraduate students. Previous studies revealed that as problem-solving skills appraisal decreased (i.e., weaker belief in ability to solve problems, higher tendency to avoid rather than confront problems, and external control of emotion), suicidal ideation and depression considerably increased (Becker-Weidman et al. (2010); Bell \&D’Zurilla (2009).

'Problem-solving skills appraisal' is an individual's perception about one's problem-solving style and the identification of one's abilities and skills to solve problems as defined by D’Zurilla\&Nezu (2007). The appraisal of problem-solving skills comprises three styles: Problem-solving confidence, Approach-avoidance style and Personal control of emotion. Problem-solving confidence is defined as having self-assurance to face a wide range of problems and trusting in one's own ability to solve the problems. The approach-avoidance style is a tendency to approach or avoid facing problems. Personal control of emotion is one's ability to control his or her emotions and behaviour while facing problems (Heppner et al., 2004). Klionsky et al. (2012) stated in their study that ineffective problem-solving abilities were associated with suicidal ideation.

The inconsistent or conflicting findings regarding relations between facets of cognitive functioning and suicide risk may indicate that there are intervening variables that influence these relations. A recent theory, the interpersonal theory of suicide (Joiner, 2005; Van Orden et al., 2010), provides variables that may explain the inconsistencies in terms of cognitive functioning and suicide and therefore may intervene in relations between domains of cognitive functioning and suicide risk. Joiner (2005) proposed two variables that, in combination, create suicide ideation: perceived burdensomeness and thwarted belongingness.

Perceived burdensomeness involves the belief that one is such a burden or liability on other people that his or her death is worth more than his or her life (Joiner, 2005). Perceived burdensomeness may arise from distress due to negative life events (e.g., family conflict, medical illnesses) and feelings of expendability (Van Orden et al., 2010). Feelings of liability inherent in perceived burdensomeness are also associated with self-hatred, shame, and low self- 
esteem (Van Orden et al., 2010). A critical component of perceived burdensomeness is that other people do not have to feel burdened; it is the perception that one is a liability or burden that increases risk for suicide (Van Orden et al., 2010). In college students, perceived burdensomeness has been consistently linked to suicide ideation (Lamis, Leenaars, Jahn, \& Lester, 2013; Van Orden et al., 2008; Zhang et al., 2013).

Additionally, the relation between depressive symptoms and suicide ideation was mediated by perceived burdensomeness in college students (Davidson, Wingate, Grant, Judah, \& Mills, 2011), similar to the findings in older adults, indicating that perceived burdensomeness is a proximal risk factor for suicide ideation. The aim of the current study was to examine the relationships between problem- solving skills appraisal, perceived burdensomeness, and suicidal ideation among college students. In addition, it was hypothesized that problem-solving skills appraisal (including the three components of problem-solving confidence, approach-avoidance style, and personal control of emotion) and perceived burdensomeness will be the predictors of suicidal ideation

\section{METHOD}

\section{Participants}

The sample of 350 undergraduates and postgraduates students was randomly selected from six colleges of Delhi and National Capital Region (NCR) from arts, science, commerce, and humanities stream. The participants mean age was 20.41 years with $\mathrm{SD}=1.62,49 \%$ were female and $51 \%$ were male with age ranged from 18-24 years. All participants received information sheets stating the purpose of the study and signed consent forms prior to participating in the study.

\section{MEASURES}

Problem-Solving Inventory (Heppner, 1988) The problem-solving inventory comprises 35 items that measure the perceptions of one's problem solving beliefs and style in facing problems and difficulties in one's daily life. All questions were based on a 6-point Likert scale from 1 (strongly agree) to 6 (strongly disagree). This questionnaire consists of three factors: (a) Problem solving confidence (PSC), (b) Approach-Avoidance Style; (c) Personal control of emotion. The problemsolving inventory provides scores on three problem-solving dimensions therefore, in this study, three factors were evaluated separately. The present study showed a good convergent validity for PSC, AAS, and PCE with (AVE: 0.58, 0.53, and 0.51 respectively), and the construct reliability (CR: 0.77, 0.74, and 0.71, respectively).

Interpersonal Needs Questionnaire (INQ; Van Orden et al., 2008). The INQ is an 18-item selfreport measure designed to measure the construct of perceived burdensomeness and thwarted belongingness. Items are rated on a 7 point response scale ranging from 1 (not true for me) to 7 (very true for me). Participants rate the items according to how they have felt recently. Nine items pertain to each construct and eight items are reverse scored. Mean scores are calculated for 
each construct can range from 1 to 7 , with high scores indicating higher levels of thwarted belongingness or perceived burdensomeness. Van Orden, Witte, Gorden, et al. (2008) reported good estimates of internal consistency for the thwarted belongingness (alpha $=.85$ ) and perceived burdensomeness (alpha $=.89$ ) item they reported moderate correlation in the expected directions with measure of suicidality and depressive symptoms, Reliability was also found to be good in the current sample, with Cronbach's alpha of .92 for thwarted belongingness and .89for perceived burdensomeness.

Beck Scale for Suicide Ideation (Beck \& Steer, 1991). The Beck’s Scale is a 21 item self -report instrument. The first 19 items consist of three options graded according to the intensity of the suicidality and rated on a 3 point Scale ranging from 0 to 2 . These rating are then summed to yield a total score, which ranges from 0 to 38 . The BSI is composed on three factors: desire for death (5 items), preparation for suicide (7 items), and actual suicide desire (4 items). Two BSI items, deterrents to death and deception or concealment, did not load on any factor. The BSI takes approximately 10 minutes to administer. The BSI is highly correlated with the clinically rated SSI with correlation coefficients ranging from .90 for psychiatric inpatients to .94 for outpatients. The internal reliability, test-retest stability and concurrent validity of this measure have been established.

Demographics. A self- report questionnaire was provided to obtain demographic information, such as gender, age, race, educational levels, religious affiliation, and marital status. Written informed consent was obtained from the participants in this study for their involvement in the research. This included the purpose of the research and the fact that their participation was voluntary.

\section{Procedure}

The permission was obtained for gathering data from college authority. The college students were categorized into three fields (science, social science, and Arts). Then, the student were chosen randomly from each field. After that, four classes (first, second, third, and last year undergraduate and postgraduate students) from each faculty were randomly selected, and data was collected during one of the regularly scheduled classes. The inclusion criteria for the participants were that (a) they should be college students and (b) between the age of 16 and 24 years. All the questionnaires were distributed among students. Each set of questionnaires contained an introductory letter about the aims of the study and three questionnaires (one of them was a demographic questionnaire). A total of 350 questionnaires were distributed among undergraduate and post graduate students.

\section{RESULTS}

Pearson's product moment correlations were used to assess the relationships between all the variables in the model including the Suicidal Ideation, Perceived Burdensomeness, Problem Solving Confidence, Personal Control, Approach- Avoidance style. Stepwise regression analysis was conducted to test the hypotheses. SPSS 21 Version was used to analyze the data of the study. 
Problem Solving Style, Perceived Burdensomeness and Suicide Ideation among College Students

Table 1 showing Mean, Standard Deviation and Bivariate Correlations of all the variables of the study

\begin{tabular}{|l|c|c|c|c|c|}
\hline Variables & $\begin{array}{l}\text { Suicidal } \\
\text { Ideation }\end{array}$ & $\begin{array}{l}\text { Perceived } \\
\text { Burden } \\
\text {-someness }\end{array}$ & $\begin{array}{l}\text { Problem } \\
\text { SolvingCon } \\
\text { fidence }\end{array}$ & $\begin{array}{l}\text { Personal } \\
\text { Control }\end{array}$ & $\begin{array}{l}\text { Approach- } \\
\text { Avoidance style }\end{array}$ \\
\hline Suicidal Ideation & 1 & $.500^{* *}$ & $-.352^{* *}$ & $-.391^{* *}$ & $-.522^{* *}$ \\
\hline Perceived Burdensomeness & & 1 &.$-512^{* *}$ & $-.641^{* *}$ & $-.284^{* *}$ \\
\hline Problem Solving Confidence & & & 1 & $.781^{* *}$ & $.723^{* *}$ \\
\hline Personal Control & & & & 1 & $.580^{* *}$ \\
\hline Approach-Avoidance style & & & & & 1 \\
\hline Mean & 13.98 & 15.96 & 40.63 & 19.53 & 62.08 \\
\hline SD & 5.13 & 6.89 & 11.04 & 3.45 & 17.38 \\
\hline
\end{tabular}

** Significant at $<.01$ level (2 tailed)

Table 1 summarizes the Pearson's correlations between Suicidal ideation and other variables in the study. The results indicate that significant correlations were obtained between Suicidal ideation and other variables viz. Suicidal ideation and Perceived Burdensomeness $(r=.50$, $\mathrm{p}<.001)$, Suicidal ideation and Problem Solving Confidence $(\mathrm{r}=-.35, \mathrm{p}<.001)$, Suicidal ideation and personal control $(\mathrm{r}=-.39, \mathrm{p}<.001)$, Suicidal ideation and approach -avoidance style $(\mathrm{r}=-.52$, $\mathrm{p}<.001)$.

Table 2 showing Stepwise Regression Analysis to predict suicidal ideation by ApproachAvoidance style, Perceived Burdensomeness and Problem Solving Confidence

\begin{tabular}{|c|c|c|c|c|l|}
\hline $\begin{array}{c}\text { Predicting } \\
\text { Variable }\end{array}$ & $\mathbf{R}^{2}$ & $\mathbf{R}^{2 \Delta}$ & $\boldsymbol{\beta}$ & $\mathbf{t}$ & $\mathbf{F}$ \\
\hline AAS & .272 & .272 & -.650 & -.11 .29 & \\
\hline PSC & .405 & .133 & .505 & 10.85 & $97.57^{* * *}$ \\
\hline PB & .454 & .053 & .376 & 5.82 & \\
\hline
\end{tabular}

Note: *** Significant at $<.001$ level, AAS= Approach-Avoidance style, $\mathrm{PSC}=$ Problem Solving Confidence, $\mathrm{PB}=$ Perceived Burdensomeness

Table 2 presents stepwise regression analysis. Results show that three predicting variants: approach -avoidance style, problem solving confidence and perceived burdensomeness entered in regression equation respectively. All variables significantly contributed to the prediction of suicidal ideation. Table shows that all the variables together contributed $45 \%$ variance in predicting suicidal ideation. Approach -avoidance style made separate significant contributions to the prediction of suicidal ideation $\left(\mathrm{R}^{2} \Delta^{=} .272, \mathrm{p}<.001\right)$, Problem Solving Confidence $\left(\mathrm{R}^{2} \Delta=.133, \mathrm{p}<.001\right)$ and Perceived Burdensomeness $\left(\mathrm{R}^{2} \Delta=.053, \mathrm{p}<.001\right)$ made 
separate significant contributions. In the present study Approach -avoidance style is found to be most potent predictor of suicidal ideation.

\section{DISCUSSION}

The current study was conducted to study the relationships between problem- solving skills appraisal, perceived burdensomeness, and suicidal ideation among college students. The positive significant relationship was found between the perceived burdensomeness and suicidal ideation among college students. The finding of the study indicates to the fact that people high on perceived burdensomeness tend to show higher suicidal ideation. Study conducted by Van Orden et al., (2008) found that desire for death results from the occurrence of a sense of perceived burdensomeness. Perceived burdensomeness has consistently been associated with suicidal ideation in a number of samples (Bryan et al., 2010; Van Orden et al., 2008).

Further it was observed from the result of the present study that suicidal ideation among student is significantly predicted by Avoidance style of handling situation, poor problem solving confidence and perceived burdensomeness. Results are consistent with prior research demonstrating a relationship between problem-solving deficits and increased suicidal behavior and also between problem-solving strengths and decreased risk for suicidal behavior From the findings it may be interpreted that students with poor confidence and perceived burdensomeness in their life report higher level of suicidal ideation (Clum \& Febbraro, 1994: Esposito \& Clum, 2002). Problem solving confidence has often been found an inverse association with suicide and some evidence suggests it may have value as a buffer against risk. A study conducted by Van Orden, Lynam, Hollar \& Joiner (2006) have recounted that perceived burdensomeness was a strong predictor of suicidal ideation even when controlling for powerful covariates such as depression and hopelessness. Poor problem-solving skills have been consistently identified as a risk factor for suicidal behavior across age groups (McAuliffe et al., 2006; McLaughlin, Miller, \& Warwick, 1996).

\section{CONCLUSION}

The research paper highlights that students reports higher suicidal ideation with lack of problem solving confidence, poor emotional control and feeling of perceived burdensomeness. Approach - avoidance style emerged to be a much potent variable to predict to suicidal ideation.

\section{IMPLICATION}

The present study demonstrated that how poor problem solving skill and feeling of burdensomeness affects suicidal ideation among students. The study suggests counseling and intervention should be started in the colleges to provide adequate guidance in order to face stressful situations. Situation reaction test can help in identifying students having limited problem solving ability. As far as possible sufficient opportunity should be given to maximum students in organizing various activities even at class room level to help them learn taking 
decisions and face unfamiliar situations. Case studies on successful individuals within their field of study should be introduced highlighting, initial failures and their persistence towards the goal leading to success.

\section{REFERENCE}

Beck, A. T., Steer, R. A., \&Ranieri, W. F. (1988). Scale for suicide ideation: Psychometric properties of a self-report version. Journal of clinical psychology. 44(4), 499-505.

Becker-Weidman, E. G., Jacobs, R. H., Reinecke, M. A., Silva, S. G., \& March, J. S. (2010) Social problem-solving among adolescents treated for depression. Behaviour Research and Therapy, 48(1), 11-18.

Bell, A. C., \&D’Zurilla, T. J. (2009). Problem-solving therapy for depression: A meta-analysis. Clinical Psychology Review 29, 348-353.

Bryan, C. J., Morrow, C., Anestis, M., \& Joiner, T. (2010). A preliminary test of the interpersonal-psychological theory of suicidal behavior in a military sample. Personality and Individual Differences, 48, 347-350.

D’Zurilla, T. J., \&Nezu, A. M. (2007). Problem-solving therapy: A positive approach to clinical intervention. (3rd ed.). New York: Spring Publishing Company.

Esposito, C. L.,\& Clum, G. A. (2002). Social support and problem-solving as moderators of the relationship between childhood abuse and suicidality: applications to a delinquent population. Journal of Trauma Stress, 15(2), 137-146.

Garlow, S. J., Rosenberg, J., Moore, J. D., Haas, A. P., Koestner, B., Hendin, H. \&Nemeroff, C. B. (2008). Depression, desperation, and suicidal ideation in college students: results from the American Foundation for Suicide Prevention College Screening Project at Emory University. Depression and Anxiety 25(6)482-488.

Heppner, M. J., Lee, D., Paul Heppner, P., McKinnon, L. C., Multon, K. D., Gysbers, N. C. (2004). The role of problem-solving appraisal in the process and outcome of career counseling. Journal of Vocational Behavior, 65, 217-238.

Heppner, P. P. (1988). The problem solving inventory: Manual. Palo Alto, CA: Consulting Psychologists Press.

Kimberly, A., Van Orden, Tracy, K., Witte, Kelly, C., Cukrowicz, Scott Braithwaite, Edward A. Selby., \& Thomas. E. Joiner, Jr. (2010). The Interpersonal Theory of Suicide. Psychological Review, 117(2), 575-600.

Klionsky, D. J., Abdalla, F. C., Abeliovich, H., Abraham. R. T., Acevedo-Arozena, A., Adeli, K., et al. (2012) Guidelines for the use and interpretation of assays for monitoring autophagy. Autophagy, 8(4), 445-544.

Lamis, D. A., Leenaars, L. S., Jahn, D. R. \& Lester, D.(2013). Intimate partner violence: are perpetrators also victims and are they more likely to experience suicide ideation?, Journal of Interpersonal violence, 28(16), 3109-3128.

McAuliffe, C., Corcoran, P., Keeley, H., Arensman, E., Bille-Brahe, U., de Leo, D., et al. (2006). Problem-solving ability and repetition of deliberate self-harm: A multicentre study. 
Psychological Medicine: A Journal of Research in Psychiatry and the Allied Sciences, 36(1), 45-55.

McLaughlin, J., Miller, P., \& Warwick, H. (1996). Deliberate self-harm in adolescents: Hopelessness, depression, problems and problem-solving. Journal of Adolescence, 19(6), 523-532.

National Center for Injury Prevention and Control (2010) National Center for Injury Prevention and Control. Available: www.cdc.gov/ncipc/wisqars.

Van Orden, K. A., Witte, T., Gordon, K., Bender, T., \& Joiner, T. (2008). Suicidal desire and the capability for suicide: Tests of the interpersonal-psychological theory of suicidal behavior among adults. Journal of Consulting and Clinical Psychology, 76, 72-83.

World Health Organization (2012) Suicide prevention (SUPRE). \Available: http://www.who.int/mental_ health/prevention/suicide/suicideprevent/en/index.html

Zhang, Y., Ling, J., Yuan, C., Dubruille, R., Emery, P. (2013). A role for Drosophila ATX2 in activation of PER translation and circadian behavior. Science,340(6134), 879-882.

How to cite this article: A Gautam (2016), Problem Solving Style, Perceived Burdensomeness and Suicide Ideation among College Students, International Journal of Indian Psychology, Volume 3, Issue 3, No. 10, DIP: 18.01.172/20160303, ISBN: 978-1-365-19879-3 